\title{
Interference of nonpolarized light in liquid crystal domains on the polymer surface
}

\author{
Alexander M. Parshin, ${ }^{1,2,{ }^{*}}$ Alexey V. Barannik, ${ }^{1,2}$, Victor Y. \\ ZYRYANOV $^{1}$ AND VASILY F. SHABANOV ${ }^{1}$ \\ ${ }^{1}$ Kirensky Institute of Physics, Krasnoyarsk Scientific Center, Siberian Branch, Russian Academy of \\ Sciences, Krasnoyarsk, 660036 Russia \\ ${ }^{2}$ Siberian Federal University, Krasnoyarsk, 660041 Russia \\ *parshin@iph.krasn.ru
}

\begin{abstract}
The interference of the nonpolarized light transmitted through an ensemble of nematic liquid crystal domains formed on the polycarbonate surface has been investigated. A model based on the superposition of the ordinary and extraordinary beams passed through domains with the radial structure has been developed. Expressions for the phase difference and intensity of the interfering beams, which take into account the distribution of the liquid crystal director field, have been derived. The dependences of the optical transmittance of a domain layer on the applied voltage have been calculated with regard to the material and optical constants of a liquid crystal and structural features and averaged morphological parameters of individual domains in the experimental sample. The results of the calculation are consistent with the experimental data, which confirms the validity of the proposed model.
\end{abstract}

(c) 2018 Optical Society of America under the terms of the OSA Open Access Publishing Agreement

\section{Introduction}

In classical experiments, the interference of nonpolarized light beams occurs via dividing the wave front or amplitude [1]. Under these conditions, coherent waves with the oscillations in all possible directions with the same initial phase meet. In this case, the waves polarized perpendicular to each other do not interfere and the waves with the same oscillation direction form a developed interference pattern. On the other hand, the interference phenomena are observed in crystals with the optical axes. The light beam entering a crystal plate at an angle to the optical axis is divided into two beams, ordinary and extraordinary, with different refractive indices. If the beams are neither scattered nor transformed by the polarizers, then, after leaving a crystal, they do not interfere, being polarized in the mutually perpendicular directions. Only placing a crystal plate between two polarizers, one can separate the beams oscillating in the same plane and obtain the interference in parallel beams, which depends on the phase difference between polarized components. In liquid crystals (LCs), the birefringence-induced interference is also found. The interference effects are observed most frequently in uniformly aligned nematic LC layers with the optical axis coinciding with the director. Placing the LC layer between two polarizers, one can obtain the interference oscillations with the light intensity maxima and minima by changing the phase difference between the ordinary and extraordinary beams using an external electric or magnetic field under the conditions of the Frederiks transition [2]. In the misoriented LC layers, the birefringence effects can be observed without polarizers [3-5]. In [3], the interference maxima and minima were found using the monochromatic light. The authors attributed the oscillatory character of the electric field dependences of the optical transmittance to the selective scattering of light on randomly formed LC domains in the framework of the Mie theory [6]. According to this theory, the oscillations are characterized by the phase shift between the beams that passed through a domain and beyond it. Similar dependences were 
observed in studying the light propagation through nematic LC droplets dispersed in polymer matrices [7-11] with the analogous data interpretation. Later, the light intensity oscillations in an electric or magnetic field were observed when transmitting the laser radiation through ensembles of nematic LC domains with the radial structure on the polycarbonate (PC) surface $[12,13]$. The interference effects in these structures could not be explained using the Mie theory because of the large size and close packing of domains. In addition, it was shown [14] that the oscillatory character of the dependences is caused by the configuration of the LC director in an individual domain. Light intensity oscillations in [12-14] were interpreted only qualitatively using the gradient optics approach [15]. These previous studies were performed with the use of partially polarized laser radiation, and it was not excluded the possibility of decisive influence of the polarization on the interference oscillations. The aim of this article is to study the interference of nonpolarized light propagating through LC domains on the PC surface as a function of the electric field with the use of the variation method for minimizing the free energy and calculating the optical characteristics with regard to the scattering.

\section{Model}

We consider the propagation of nonpolarized light with the oscillations in all directions through an individual domain in the ensemble. In the initial approximation, we assume a domain to be a cylinder located between cell plates with voltage $U$ applied to its bases (Fig. 1). The light falls along the cell plane normal. We choose arbitrary points 1 and 2 on an imaginary plane that crosses the domain perpendicular to the incident light. Obviously, the beams transmitted without deviation do not form a superposition in spatially separated points 1 ' and $2^{\prime}$ on the photodetector plane. Under the assumption of scattering of a small amount of light passed through the domain, there is always some point $O$ on a fairly distant detector where the beam from point 1 converges with the beam from point 2. According to the continual theory, the optical properties of an LC at each point correspond to those of a uniaxial crystal [16]. In this approximation, the vicinities of the chosen points can be considered to be small crystal plates with the optical axes along the director, which make the passing beam experience the birefringence. When the optical axes are co-directed, the beams at the given points could not interfere either, since their ordinary and extraordinary components would always be polarized in the mutually perpendicular directions.

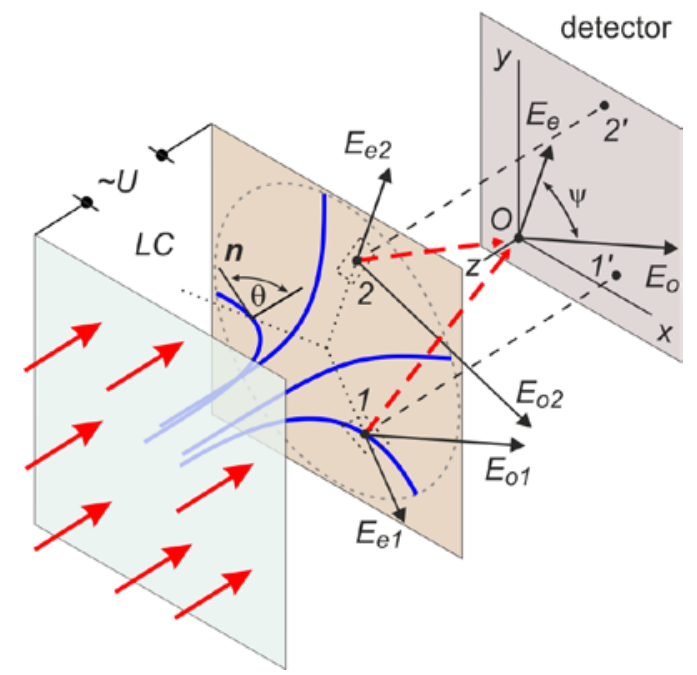

Fig. 1. Propagation of the nonpolarized light through a radially homeotropic nematic structure in a domain. The director $\boldsymbol{n}$ makes angle $\theta$ with the $z$ axis, which coincides with the direction of the incident light. The ordinary $E_{\mathrm{o}}=E_{\mathrm{o} 1}$ and extraordinary $E_{\mathrm{e}}=E_{\mathrm{e} 2}$ waves from points 1 and 2 interfere at the $O$ point of the detector. 
In the investigated scheme, the nematic forms a radial orientation structure. At each point of the chosen plane, the director $\boldsymbol{n}$ makes the same angle $\theta$ with the normal and is azimuthally oriented along the domain radius. Consequently, the extraordinary components $E_{\mathrm{e} 1}$ and $E_{\mathrm{e} 2}$ are co-directed to the corresponding radii and the ordinary components $E_{01}$ and $E_{02}$ are perpendicular to them. First, let us consider a particular case of the incident plane-polarized light from a coherent source. Then, the ordinary beam from point 1 can interfere with the extraordinary beam from point 2 at the point $O$ due to the non-perpendicular directions of the oscillations $E_{01}$ and $E_{\mathrm{e} 2}$. Obviously, this is valid also for the pair $E_{02}$ and $E_{\mathrm{e} 1}$. Let $E_{0}=E_{01}$ and $E_{\mathrm{e}}=E_{\mathrm{e} 2}$ make angle $\psi$ on the detector plane. Taking into account the phase difference $\delta$, which occurs during their propagation through a refracting medium, we sum their projections onto the corresponding axes of the Cartesian system of coordinates specified at the point $O$ and square these sums. The sum of $E_{x}^{2}$ and $E_{y}^{2}$ yields the squared amplitude of the resulting oscillation

$$
E^{2}=E_{o}^{2}+E_{e}^{2}+2 E_{o} E_{e} \cos \psi \cos \delta
$$

Taking into account that all the $\psi$ values in a uniform radial structure are equiprobable, we may expect that the interference term will appear in the general interference pattern as well. Let $n_{\mathrm{o}}$ and $n_{\mathrm{e}}$ be the nematic refractive indices for the light with wavelength $\lambda$. At any domain point, the ordinary beam propagates in the LC with the same velocity independent of the angle of incidence $[1,17]$. The extraordinary beam propagation velocity changes along the $z$ axis upon variation in the effective refractive index $n_{\text {eff, }}$ which is determined by only the angle $\theta$ of the director inclination to this axis:

$$
n_{\text {eff }}=\frac{n_{e} n_{o}}{\sqrt{n_{e}^{2} \cos ^{2} \theta+n_{o}^{2} \sin ^{2} \theta}}
$$

Since, azimuthally, the $E_{\mathrm{e}}$ component is always co-directed to the director, the refractive index $n_{\text {eff }}$ is the same over the entire plane specified by the $z$ coordinate. The phase difference at the trend of the nematic director $\boldsymbol{n}$ to pass in an electric field from the radial-planar orientation on the PC surface, where $\theta=\pi / 2$, to the homeotropic orientation $\theta=0$ in the bulk layer is determined as

$$
\Delta=\frac{2 \pi}{\lambda} \int_{0}^{\xi_{E}}\left(n_{e f f}(z)-n_{o}\right) d z
$$

where $\xi_{\mathrm{E}}$ is the electric coherence length [16].

We write the free energy in the presence of electric field $\boldsymbol{E}$ acting on the LC in volume $V$ in the form

$$
F=\frac{K}{2} \int_{V}\left[(\nabla \cdot \boldsymbol{n})^{2}+(\nabla \times \boldsymbol{n})^{2}-\frac{1}{8 \pi} \Delta \varepsilon(\boldsymbol{n} \cdot \boldsymbol{E})^{2}\right] d V,
$$

where $K$ is the modulus of elasticity, $\Delta \varepsilon$ is the permittivity anisotropy. In this expression, two simplifications were made. First, the modulus of elasticity is presented in the one-constant approximation $K=\left(K_{11}+K_{22}+K_{33}\right) / 2$ [16], where $K_{11}, K_{22}$, and $K_{33}$ are the moduli of elasticity for the splay, twist and bend distortions, respectively. The estimation of the effect of this approximation on the free energy made in [19] showed satisfactory agreement between the experiment and theoretical consideration. Second, the electric field depends on $z$, so the exact expression should contain, instead of $\boldsymbol{E}$, the factor $4 \pi D_{z} /\left(\varepsilon_{\perp} \sin ^{2} \theta+\varepsilon_{\|} \cos ^{2} \theta\right)$, where $\varepsilon_{\perp}$ and $\varepsilon_{\|}$are the permittivities parallel and perpendicular to the LC directors, respectively, and $D_{z}$ is the electric displacement, which is constant along the $z$ axis. Nevertheless, the estimation based on the comparison of the voltage and magnetic field dependences of the 
light intensities made by us in [12] for the similar structure showed that the error introduced by this approximation should not be significant.

According to the configuration shown in Fig. 1, we express the components of the director $\boldsymbol{n}$ in the cylindrical coordinates: $n_{\rho}=-\sin \theta, n_{\varphi}=0$, and $n_{z}=\cos \theta$, where $\varphi$ is the angle in the plane perpendicular to the $z$ axis [18]. The standard free energy minimization procedure yields

$$
\frac{\partial^{2} \theta}{\partial \rho^{2}}+\frac{1}{\rho} \frac{\partial \theta}{\partial \rho}+\frac{\partial^{2} \theta}{\partial z^{2}}=\left(\frac{1}{\rho^{2}}+\frac{1}{\xi_{E}^{2}}\right) \sin \theta \cos \theta
$$

When the director field is uniform along the domain radius $\rho$, the first two terms in Eq. (5) vanish and the obtained differential equation can be solved by integrating it after multiplying by $\partial \theta / \partial z$

$$
\frac{1}{\sqrt{\frac{1}{\rho^{2}}+\frac{1}{\xi_{E}^{2}}}}\left(\frac{\partial \theta}{\partial z}\right)^{2}=\sin ^{2} \theta+C
$$

On the domain surface at $\mathrm{z}=0$, the director is parallel to the domain radii $\rho$ and the angle is $\theta=\pi / 2$. In an electric field at $\mathrm{z}>\zeta_{E}$, we may assume that $\theta=0$ and $\partial \theta / \partial \mathrm{z}=0$ if the homeotropic orientation is specified on the opposite surface at $\mathrm{z}=d$. Therefore, the constant $\mathrm{C}$ will vanish and we will obtain the expression

$$
\frac{\partial \theta}{\partial z}=-\sqrt{\frac{1}{\rho^{2}}+\frac{1}{\xi_{E}^{2}}} \sin \theta
$$

Substituting $n_{\text {eff }}(z)$ from Eq. (2) and $d z$ from Eq. (7) into Eq. (3), we arrive at the relation

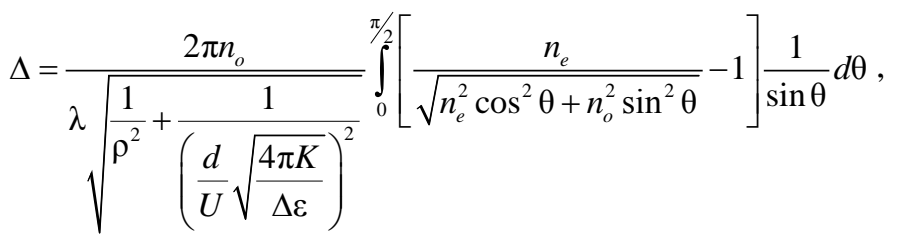

where $d$ is the domain thickness and $U$ is the voltage, which was used as $E \cdot d$ in the framework of the above simplification.

As we showed above, the velocity of propagation of the $E_{\mathrm{o}}$ and $E_{\mathrm{e}}$ waves along a domain is independent of the azimuthal director orientation. Therefore, the phase difference $\Delta$ that occurred between them during passage of the light with a certain $\lambda$ value though a domain is the same. Consequently, the equiprobable nonorthogonal interactions of all the ordinary and extraordinary components are coherent at any polarization direction of the incident beam. It can be easily shown that, due to the uniformity of the radial domain structure, points 1 and 2 specified in the cross-section plane correspond to the lines forming two intersecting diameters. Hence, in the calculation of the detected intensity $I=\left\langle E^{2}\right\rangle$, the results of superposition of the waves corresponding to all arbitrary points in the domain cross-section plane are equivalent relative to the domain rotation around the symmetry axis by an angle multiple to $\pi / 2$. Generalizing Eqs. (1) and (8) to the case of the nonpolarized light $\left\langle E_{o}^{2}\right\rangle=\left\langle E_{e}^{2}\right\rangle=I_{0} / 2[1]$ and averaging $\cos \psi$, we obtain

$$
I=I_{0}\left(1+\frac{2}{\pi} \cos \Delta\right),
$$


where $I_{0}$ is the intensity of incident light. Thus, we may expect the interference minima and maxima in the electrooptic response $I(U)$ upon sequential attaining of the $\Delta(U)$ values multiple to $\pi$ due to the rearrangement of the nematic orientation structure in a domain under the action of the electric field $[12,13]$.

\section{Experimental}

The electrooptic cell was formed from two glass plates with the transparent ITO coating. One of the plates was coated with a polymer film by centrifuging the 2-\% PC solution in dichloromethane and the other plate was treated with lecithin to specify the homeotropic orientation. A capillary gap between the plates was ensured by $30-\mu \mathrm{m}$-thick teflon gaskets and filled with the 4-n-pentyl-4'-cyanobiphenyl (5CB) LC in the nematic phase. The orientation structure of the nematic in the cell was studied $[13,18]$ on an Olympus BX51 polarization microscope (Japan). When necessary, an ac voltage with a frequency of $1 \mathrm{kHz}$ was applied to the cell electrodes.

The optical properties of the experimental samples were investigated at different voltages in the collimated light of a halogen lamp. A band with the maximum at a wavelength of $\lambda=0.630 \mu \mathrm{m}$ was separated from the lamp spectrum using light filters. A transmitted beam part scattered at angles of more than $0.5^{\circ}$ was cut by a diaphragm $3 \mathrm{~mm}$ in diameter. The intensity was detected by a Hamamatsu $\mathrm{H} 9307$ photomultiplier (Japan). All the experiments were carried out at a temperature of $t=25^{\circ} \mathrm{C}$.

\section{Results and discussion}

Figure 2 shows texture patterns of a fragment of the sample placed between crossed microscope polarizers. The pattern obtained in zero voltage is typical of a radial-planar nematic orientation, which is extended from the PC film deep in the layer by the coherence length $\xi$. Such a structure occurs whenever the LC thickness $d$ exceeds the $\xi$ value [12, 14, $18,19]$, even when the opposite cell surface was specially treated to form a homeotropic orientation. In the applied voltage $U$ higher than the threshold value $U_{\mathrm{th}}$, at which the electric coherence length $\xi_{\mathrm{E}}$ becomes equal to $\xi$ [14], the texture acquires the form characteristic of a radial-homeotropic structure (Fig. 2, in the bottom). It can be seen that, under the action of an electric field, the domains and their sectors change their color. However, one can see that the color does not change along the domain radii, which is indicative of the uniform distribution of the director tilt. This allows us to make an assumption $\partial \theta / \partial \rho=0$ for the chosen structure preparation technique and interpret the results using simplified relation (7). 

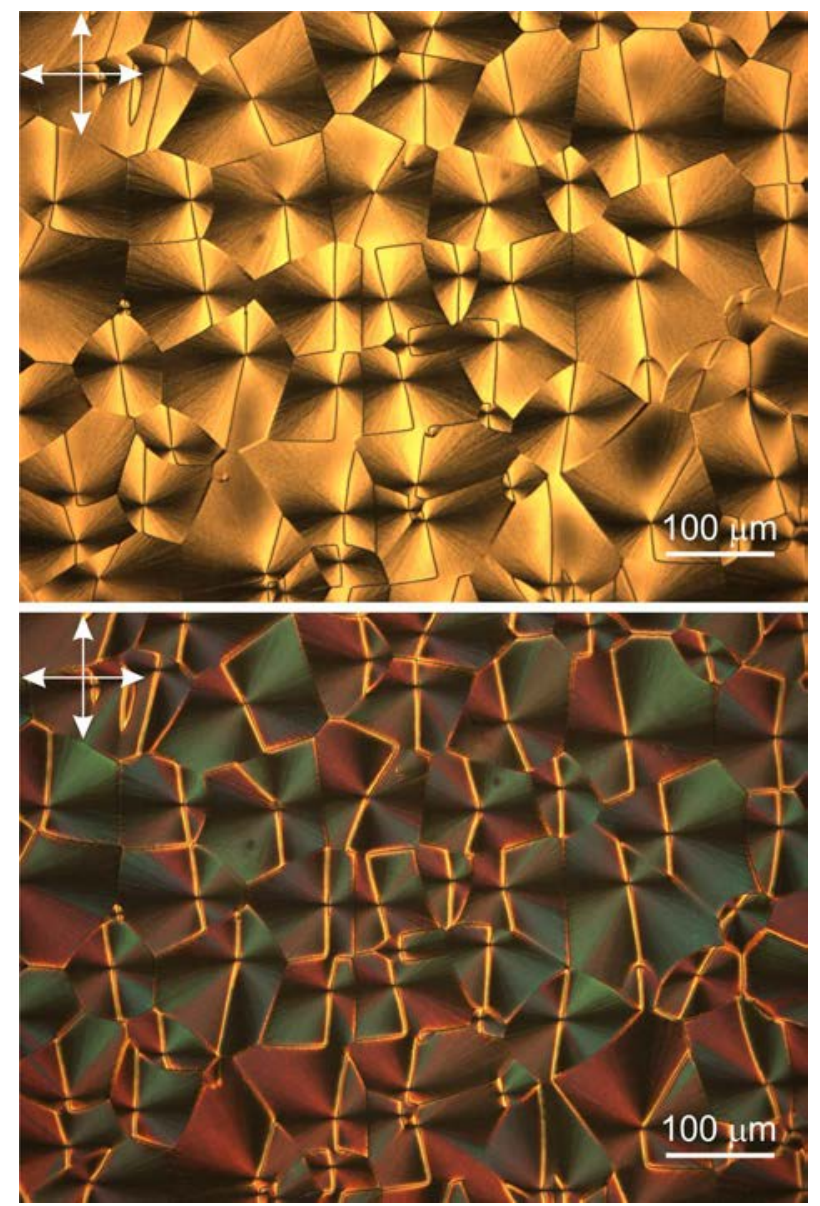

Fig. 2. Microphotograph of an ensemble of domains formed in a plane cell with one plate coated with the PC film (on the top). The change in the texture pattern in the photograph in the bottom is caused by an applied voltage of $U=4.65 \mathrm{~V}$ Arrows show the directions of microscope polarizers.

Figure 3 shows the experimental and calculated (Eqs. (8) and (9)) intensities $I$ of the light transmitted through the cell as functions of the applied voltage $U$. The electrooptic response curve $I(U)$ contains several extrema above the threshold value $U_{\mathrm{th}}$, which are followed by the smooth saturation. In most investigated samples, the contrast as a ratio between the intensities of the transmitted light in the neighboring interference maxima and minima attained 5 . In the calculation, we used the following literature data for the $5 \mathrm{CB}$ nematic at $t=25^{\circ} \mathrm{C}$ : $n_{\mathrm{e}}=1.7057, n_{\mathrm{o}}=1.5281$ [20], $K=5.48 \cdot 10^{-7}$ dyn, and $\Delta \varepsilon=13.3$ [21]. The voltage $U$ was varied with a step of $0.01 \mathrm{~V}$, starting with a threshold value of $U_{\text {th }}=0.4 \mathrm{~V}$, which was determined from the equalities $\xi_{\mathrm{E}}=(4 \pi K / \Delta \varepsilon)^{1 / 2} d / U$ and $\xi=r /[(1-\pi / 4+\pi / 12) / 2 \pi / \ln (r / b)]^{1 / 2}$ $[12,18]$, where $d=30 \mu \mathrm{m}$ is the LC layer thickness, $b=10 \mu \mathrm{m}$ is the disclination line width, and $\rho=r=50 \mu \mathrm{m}$ is the average domain radius (Fig. 2). To fit the measured data to the calculated ones, we used correction coefficients that take into account the loss to the reflection by the cell substrate surfaces and scattering at the LC-polymer interface and domain walls and disclination lines in domains $[13,18,19]$. As a result, Eq. (9) takes the form $I=A+B(1+2 / \pi \cdot \cos \Delta)$. 


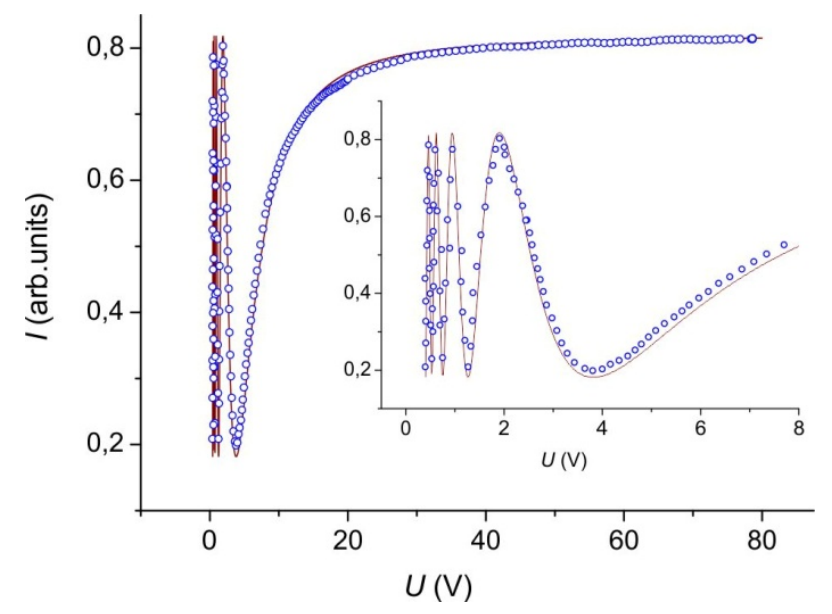

Fig. 3. Experimental (circles) and calculated (solid line) intensities $I$ of the light with a wavelength of $\lambda=0.630 \mu \mathrm{m}$ passed through an ensemble of LC domains as functions of voltage $U$. Inset: initial portion of the light transmittance below $5 \mathrm{~V}$.

In addition, to study the scattering, the glass plates without polymer coating and with the PC film and the filled LC cell were alternatively probed. The observed minor scattering on the PC-coated substrate is most likely caused by the diffraction of light on structural inhomogeneities developed in the surface layer during fast evaporation of a solvent [22] and is comparable with the scattering on rough polymer surfaces [23]. Upon embedding the LC into a plane cell capillary, the scattering increased for a short time, which was apparently caused by the nematic layer nonuniformity. The transmitted light intensity that was set or several seconds starts slowly decreasing according to the exponential law (Fig. 4). Simultaneously, domains arise and grow on the PC surface. The process lasts for a few seconds and is characterized by the domain radius growth linear in time. It follows from the data presented in Fig. 4 that the light transmittance changes monotonically during the domain structure formation. Thus, we may state that the characteristic interference form of the curves in Fig. 3 is caused by the independent contribution of each domain to the general pattern of the electrooptic response and not related to the domain structure morphology in an ensemble.

It is worth noting that, at the comparison of the results, the account for the dispersion in the medium was simplified. This approach is justified by the fact that the contribution of the wavelength to the phase delay as compared with the contribution of the control voltage is much lower in the order of magnitude. In Eq. (8), the variables are related as $\lambda \sim U \cdot 10^{-5}$ in the CGSE units. In particular, the electrooptic response curve obtained experimentally without using light filters had the similar form. The qualitative estimation by Eq. (8) show also that the contribution of the dispersion to the phase delay at $\rho>>\lambda$ is insignificant. The results of 


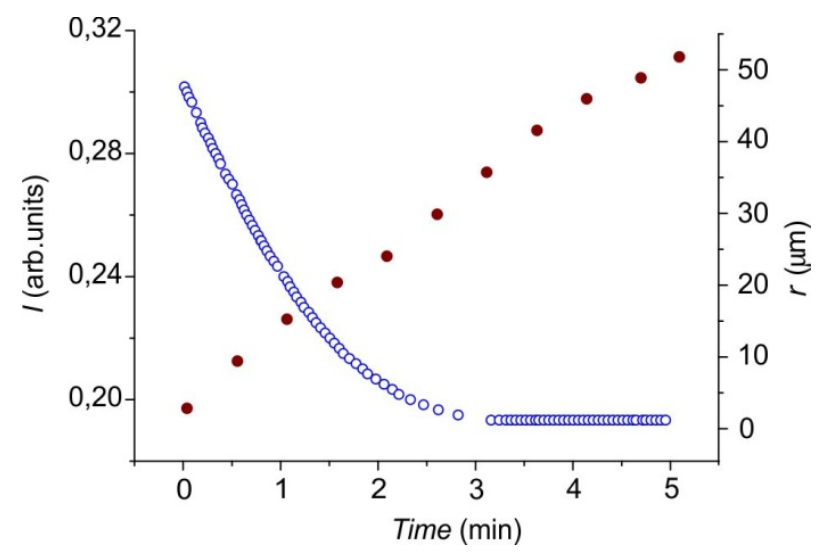

Fig. 4. Variation in intensity $I$ of the light transmitted through the sample (left-hand axis) and average domain radius $r$ (right-hand axis) during the LC domain structure formation on the PC film.

the calculation with the varied parameters show that only when the domain radius decreases below $\rho<5 \mu \mathrm{m}$, the extrema in the $I(U)$ curve start slowly shifting to the left. In addition, the model used does not take into account the real non-cylindrical form of individual domains and domain structure morphology. Nevertheless, good agreement between the data calculated using the above-made simplifications and experimental values confirms the correctness of the theoretical approach.

\section{Conclusions}

The propagation of the nonpolarized light through an individual nematic LC domain in their ensemble in the applied voltage was investigated theoretically and experimentally. We developed a model based on the superposition of the ordinary and extraordinary beams passed through the domain with the radial structure and experimentally confirmed the validity of the model. For the light intensity $I$, the equation including the phase difference $\Delta$ between the extraordinary and ordinary beams was derived as well as the relation between the $\Delta$ value and voltage $U$ considering the material and optical LC parameters. The electrooptic cell formed from two plates with ITO, one being coated with the PC layer, was filled with the 5CB nematic. The texture patterns of the domain ensemble were examined using a polarization microscope. It was demonstrated that, at the transition of the nematic orientation structure from radial-planar on the PC surface to homeotropic in the bulk of a domain under the action of an applied voltage, the structural nonuniformity is retained. The light transmittance was measured using a halogen lamp as a light source. The calculated and experimental $I(U)$ dependences were found to be consistent. The extrema observed experimentally in the electrooptic response were explained by the interference caused by the phase shift between the nonorthogonal ordinary and extraordinary waves. This effect results from the phase splitting of the wave front of a birefringent domain structure of a domain with the radial LC director orientation. The analysis of the light scattering characteristics of the LC cell during the formation of domains in the nematic layer showed no interrelation between the morphological structure of the entire ensemble and interference character of the electrooptic response. Using the proposed model, we qualitatively estimated the contribution of the dispersion and voltage to the phase delay and, consequently, to the optical transmittance. In the investigated sample, at a voltage of $U<1 \mathrm{~V}$, the switching between several states of the minimum and maximum transmittance of the nonpolarized light occurs in the band with a maximum at $\lambda=0.630 \mu \mathrm{m}$.

\section{References}

1. M. Born, E. Wolf, Principles of optics (Cambridge University Press, 1999). 
2. L. M. Blinov, V. G. Chigrinov, Electrooptic effects in liquid crystal materials (New York, Springer-Verlag, 1996).

3. O. Hotra, I. Lopatyanscyj, and B. Yavorskyj, "New electro-optical effect in nematic liquid crystal for integrated optics elements,” Proc. SPIE 5124, 112-114 (2003).

4. N. Aryasova, A. Iljin, V. Reshetnyak, Yu. Reznikov, A. Glushchenko, and J. West, "Orientation of liquid crystals on random anchoring surface,” Mol. Cryst. Liq. Cryst. 375, 165-173 (2002).

5. N. Aryasova, Yu. Reznikov, and V. Reshetnyak, "Alignment of nematic liquid crystal on the surface with spatial distribution of easy axis and anchoring energy,” Mol. Cryst. Liq. Cryst. 412, 351-359 (2004).

6. $\quad$ H. C. van de Hulst, Light Scattering by Small Particles (New York, 1981).

7. A. V. Barannik, V. F. Shabanov, V. Ya. Zyryanov, V. I. Lapanik, and V. S. Bezborodov, "Interference and ion effects in the electro-optical response of PDNLC films,” J. SID 13/4, 1-7 (2005).

8. V. V. Presnyakov, V. Ya. Zyryanov, A. V. Shabanov, and S. Ya. Vetrov, "Friedericksz threshold in bipolar nematic droplets with rigidly fixed poles,” Mol. Cryst. Liq. Cryst. 329, 27-34 (1999).

9. A. V. Konkolovich, V. V. Presnyakov, V. Ya. Zyryanov, V. A. Loiko, and V. F. Shabanov, "Interference quenching of light transmitted through a monolayer film of polymer-dispersed nematic liquid crystal," JETP Lett. 71(12), 486-488 (2000).

10. O. O. Prishchepa, A. V. Shabanov, V. Ya. Zyryanov, A. M. Parshin, and V. G. Nazarov, "Friedericksz threshold field in bipolar nematic droplets with strong surface anchoring," JETP Lett. 84(11), 607-612 (2006).

11. O. O. Prishchepa, A. M. Parshin, A. V. Shabanov, and V. Ya. Zyryanov, "Magneto-optical study of Friedericksz threshold in polymer dispersed nematic liquid crystals,” Mol. Cryst. Liq. Cryst. 488(1), 309-316 (2008).

12. A. M. Parshin, V. A. Gunyakov, V. Y. Zyryanov, and V. F. Shabanov, "Electric and magnetic field-assisted orientational transitions in the ensembles of domains in a nematic liquid crystal on the polymer surface,” Int. J. Mol. Sci. 15(10), 17838-17851 (2014).

13. A. M. Parshin, V. Y. Zyryanov, and V. F. Shabanov, "Alignment of liquid crystals by polymers with residual amounts of solvents," Scientific Reports 7(342), 1-8 (2017).

14. A. M. Parshin, V. Y. Zyryanov, and V. F. Shabanov, "Light transmission of liquid crystal domains formed by polycarbonate surface,” Optical Material Express 6(9), 2841-2846 (2016).

15. O. Sova, V. Reshetnyak, T. Galstian, and K. Asatryan, "Electrically variable liquid crystal lens based on the dielectric dividing principle,” J. Opt. Soc. Am. A 32, 803-808 (2015).

16. P. G. de Gennes and J. Prost, The Physics of Liquid Crystals (Oxford, Clarendon press, 1993).

17. L. M. Blinov, Structure and Properties of Liquid Crystals (Springer, 2011).

18. A. M. Parshin, V. A. Gunyakov, V. Ya. Zyryanov, and V. F. Shabanov, "Domain structures in nematic liquid crystals on a polycarbonate surface,” Int. J. Mol. Sci. 14(8), 16303-16320 (2013).

19. A. M. Parshin, V. Ya. Zyryanov, and V. F. Shabanov, "The director field distribution with the strongly pinned alignment in nematic structures at the polymer surface,” Liq. Cryst. 42(1), 57-64 (2015).

20. J. B. Bunning, D. A. Grellin, and T. E. Faber, "The effect of molecular biaxiality on the bulk properties of some nematic liquid crystals,” Liq. Cryst. 1(1), 37-51 (1986).

21. M. J. Bradshaw, E. P. Raynes, J. D. Bunning, T. E. Faber, "The Frank constants of some nematic liquid crystals,” J. Phys. France 46(9), 1513-1520 (1985).

22. A. M. Parshin, V. Ya. Zyryanov, and V. F. Shabanov, "Structuring of the Surface Layer of Polycarbonate Film,” Polymer Science, Series C 60(1), 23-31 (2018).

23. L. Vicari, "Liquid-crystal layer between rough polymeric surfaces," J. Opt. Soc. Am. B 16(7), 1135-1138 (1999). 